\title{
LA PRESENCIA DE MOBBING Y SUS DETERMINANTES LABORALES EN PROFESORES UNIVERSITARIOS
}

\author{
Jesús Herranz-Bellido \\ Abllio Relg-Ferrer \\ Julio Cabrera-García
}

Universidad de Alicante

\section{RESUMEN}

En este artículo se analiza la presencia de mobbing en el profesorado universitanio como parte de un estudio trasversal más amplio sobre su calidad de vida, trabajo y salud. Los objetivos del estudio son tres: 1) conocer la frecuencia del mobbing en un contexto universitario, 2) examinar la asociación existente entre mobbing y la edad, el género y la categoría académica de los profesores, y 3) estudiar los mejores predictores del mobbing. Respondieron el cuestionario 252 profesores a tiempo completo, lo que ha significado una tasa de respuesta del $61,6 \%$. Nuestros resultados muestran que casi el veintitrés por ciento $(22,6 \%)$ de los profesores se sintieron victimas de mobbing. No hemos encontrado diferencias estadísticamente significativas en mobbing debidas a la edad, género o categoría académica de los profesores. Según diversos análisis de regresión logística jerárquica por bloques que hemos realizado, los mejores predictores del mobbing han resultado ser: el grado de autonomia en el trabajo y la satisfacción 
experimentada en las relaciones con los supervisores. Estas 2 variables han explicado, en nuestro estudio, casi un $37 \%$ de la variabilidad del mobbing. Serían necesarios estudios longitudinales o experimentales para poder establecer relaciones de causalidad entre mobbing y contexto laboral.

Palabras clave: MOBBING; ACOSO LABORAL PSICOLOGICO; PROFESO RES UNIVERSITARIOS; ANÁLISIS DE REGRESIÓN LOGÍSTICO; GENERO; EDAD; CATEGORIA ACADÉMICA; CALIDAD DE VIDA EN EL TRABAJO; SATISFACCIÓN LABORAL.

\section{SUMMARY}

This article examines the mobbing phenomenon among university professors. The data belong to a broader, crosssectional study on quality of life, job satisfaction and health. The three objectives of the article are: 1) to provide an estimate of the prevalence of mobbing within a university environment, 2) to examine the prevalence of mobbing in relation to the age, gender, and faculty rank, and 3) identify the best predictors of mobbing. A total of 252 full-time faculty responded to the survey, which corresponds to a $61.6 \%$ participation rate. About $23 \%$ of the professors reported having experienced mobbing. Mobbing prevalence rates did not differ significantly across ages, genders, or academic ranks. Using logistic regression methods, we found that low job autonomy and low interaction-satisfaction with supervisors were the best predictors of mabbing incidence. These two predictors explained about $37 \%$ of the mobbing variance. The findings justify the need for longitudinal investigations to assess cause-effect relationship within the job context of university professors.

KeY WOrd8: MOBBING; UNVERSTY PROFESSORS; LOCISTIC RECRESSION ANALYSIS; GENDER; AGE; ACADEMIC RANK; WORK-RELATED QUALTT OF LIFE; JOB SATSFACTION. 


\section{INTRODUCCIÓN}

Cuando se habla de mobbing se alude a un continuo y deliberado maltrato verbal y modal que recibe un trabajador por parte de otro u otros, quienes se comportan con él cruelmente, con vistas a lograr su aniquilación psicológica y a conseguir su salida de la organización laboral (Piñuel, 2001). Con el mobbing se pretende la destrucción psicológica de la victíma por medio de actuaciones más o menos hostiles que consideradas de forma aislada podrian parecer anodinas, pero cuya repetición produce efectos muy perjudiciales (Leymann, 1990). Según su direccionalidad es posible considerar tres tipos de mobbing. descendente (el que se manifiesta de superior a subordinado), horizontal (entre trabajadores de similar categoría profesional) o ascendente (de subordinado-s a superior jerárquico). En Estados Unidos el $81 \%$ de los casos de mobbing es descendente (Namie, 2000). En Europa el mobbing descendente es también el más habitual: 82\% (Piñuel, 2002a), 57\% (Quine, 1999), 54\% (Einarsen y Skogstad, 1996), 52\% (Rayner, 1998), 47\% (Kistner, 1997, cit. Vandekerckhove y Commers, 2003 -pág. 42).

Uno de los aspectos más controvertidos en el estudio del mabbing es el relacionado con su frecuencia y duración. ¿Cuándo se puede afirmar que una situación de acoso laboral debe ser considerada mobbing? Para Leyman (1990) existe mobbing cuando el acoso aparece como mínimo una vez a la semana durante un intervalo de al menos 6 meses. Este mismo criterio temporal es el utilizado en el estudio de Fidalgo y Piñuel (2004). Einarsen y Skogstad (1996) afirman que sólo debe considerarse la presencia de mobbing cuando las manifestaciones que lo caracterizan aparecen durante un periodo mínimo de 6 meses, ya sea "de vez en cuando" o "semanalmente". En la investigación llevada a cabo por Björkqvist, Osteman y HjeltBack (1994) se indagó acerca de conductas persistentes de mobbing existentes durante ef año anterior al estudio.

La presencia del mobbing varla notablemente según diferentes estudios, llegando a oscilar entre el 1 y el $38 \%$ dependiendo de la estrategia de medida utitizada, la profesión, el sector ocupacional o el país en el que se lleve a cabo el estudio (DiMartino, Hoel y Cooper, 2003). En uno de los primeros estudios en los que se analiza la prevalencia 
de mobbing, Leymann (1990) estima que un 3,5\% de los trabajadores escandinavos sufre acoso laboral psicológico, existiendo un mayor porcentaje de acosados entre trabajadores de las enseñanzas primaria, media o universitaria, trabajadores de la salud (especialmente el personal de enfermería), cuidadores de guarderías y escuelas infantiles, y miembros de organizaciones sin animo de lucro o de instituciones y organizaciones religiosas. Las cifras de prevalencia de mobbing varian notoriamente. Paolí y Merlié (2001), por ejemplo, encuentran que un $9 \%$ de trabajadores europeos reconoce haber sufrido alguna forma de violencia psicológica en el trabajo, mientras que Quine (1999) detectó una elevada prevalencia de mobbing (38\%) en personal sanitario inglés.

Diferentes investigaciones muestran que el mobbing influye negativamente en la salud, la calidad de vida y la satisfacción laboral de los trabajadores (Quine, 1999; Kivimăki et al., 2000, 2003; Paoli y Merllié, 2001; DiMartino et al., 2003; Piñuel, 2001, 2002a, 2002b).

Nuestro objetivo aquí fue, en el marco de una investigación más amplia sobre la calidad de vida, el trabajo y la salud en los profesores de la Universidad de Alicante, estimar la frecuencia del mobbing entre el profesorado universitario, y explorar sus determinantes laborales, de salud, de calidad de vida y sociodemográficos.

\section{MATERIAL Y MÉTODOS}

\section{Diseño de la Investlgaclón}

Encuesta transversal, autoinformada y administrada por correo intemo.

\section{Personas y procedimlento de recogida de datos}

Antes de contactar con el profesorado se cursó una petición a la Gerencia de la Universidad de Alicante en la que se pidió autorización para la llevar el estudio, indicándose la finalidad del mismo, la necesidad de los datos solicitados al profesorado, asf como el compromiso de confidencialidad y reserva de la información obtenida.

La población de este estudio la constituyen los 1782 profesores funcionarios y contratados de la Universidad de Alicante. Inicialmente 
fueron seleccionados 589 participantes mediante un muestreo aleatorio estratificado por categorías y con afijación no proporcional. (Error de muestreo: 0.05 . Varianza poblacional: $p=q=0.5 ; \delta=0.25$ ). Contestaron al cuestionario 331 profesores funcionarios y contratados a tiempo completo y a tiempo parcial. De éstos fueron separados, para realizar este estudio, los profesores asociados a tiempo parcial $(n=79)$, lo que ha permitido trabajar con una muestra más homogénea, desde el punto de vista ocupacional, compuesta exclusivamente por profesores con dedicación plena. La muestra final utilizada en la investigación ha sido de 252 profesores con dedicación laboral a tiempo completo.

La recogida de datos se llevó a cabo por medio de un cuestionario autocumplimentado, voluntario y anónimo, enviado a través del servicio de correo interno de la universidad a la dirección oficial del campus donde los profesores reciben su correo profesional. El cuestionario fue remitido junto con una carta de presentación que explicaba los objetivos del estudio y un sobre de correo interno para devolver el cuestionario una vez cumplimentado. Nueve dias después se envió una carta recondatorio en la que se agradecía la participación a aquellos profesores (desconocidos para los investigadores, dado el carácter anónimo del estudio) que hablan contestado en cuestionario y se solicitaba la participación a los que aún no lo habian hecho. Finalmente, a las tres semanas del primer contacto se envió una tercera y última comunicación con un nuevo ejemplar del cuestionario, un breve carta animando a su contestación, si aún no se había hecho, y un nuevo sobre de correo interno para la devolución de los datos.

\section{Instrumento de evaluación}

Se ha elaborado un cuestionario autocumplimentado de 71 preguntas para evaluar la calidad de vida, el trabajo y la salud de los profesores universitarios. Dentro de este estudio amplio se ha examinado la presencia y frecuencia del mobbing por medio de una pregunta de elaboración propia que ha presentado la siguiente formulación: Durante el último año ¿se ha sentido acosada/acosada laboralmente (maltratado, perseguido, arrinconado...) y cuatro alternativas de respuesta: Frecuentemente; Algunas veces; Casi nunca; y Nunca. La variable mobbing se ha dicotomizado: se ha puntuado 
con un 1 si se refiere mobbing (esta categoría agrupa las altemativas de respuesta Frecuentemente y $A$ veces) y con un 0 si no se refiere mobbing (esta categoría agrupa las altemativas de respuesta Nunca y Casi nunca). Las variables predictoras y los instrumentos para su medida utilizados en el estudio han sido los siguientes:

A) La calidad de vida laboral: Ha sido medida por dos sistemas de evaluación: de una parte se ha hecho una estimación del bienestar laboral. Para ello se han utilizado 10 dimensiones establecidas por Warr (1999) que valoran los siguientes aspectos laborales: oportunidad para control personal; oportunidad para la utilización de habilidades o destrezas personales; metas generadas extemamente; variedad de la tarea realizada; claridad ambiental; disponibilidad de dinero; seguridad física; supervisión de apoyo; oportunidad para las relaciones interpersonales; y posición social valorada. De otra parte, se han estudiado, también como variables predictoras del mobbing, siete índices de calidad de vida laboral procedentes de la Encuesta sobre Calidad de Vida en el Trabajo (ECVT) (MTAS, 1999, 2000, 2001, 2002). Estos indices miden las siguientes dimensiones: calidad de vida en el trabajo; condiciones de trabajo; orgullo en el trabajo; alienación en el puesto de trabajo; participación en el puesto de trabajo; integración el puesto de trabajo; y autonomía en el puesto de trabajo. Además para una más completa valoración de la dimensión laboral de los profesores hemos incluido una variable, en formato mono-ítem, que mide el grado de satisfacción laboral general y que se puntúa entre 1 (Muy insatisfecho) y 10 (Muy satisfecho). Es una varlable que, como las anteriores, procede de la ECVT (MTAS, 1999, 2000, 2001, 2002). Finalmente, se han formulado cinco cuestiones que valoran el grado de satisfacción laboral específico del profesorado con aspectos concretos de su trabajo universitario: la docencia que imparten, la investigación que realizan, las relaciones con los compañeros, las relaciones con los estudlantes, y la situación y promoción académicas. Estas preguntas son una adaptación del CESP (Cuestionario para la Evaluación de la Satisfacción del Profesorado) de Sáenz y Lorenzo (1993).

B) La salud de los profesores se ha valorado por medio de cuatro variables: una medida monoitem de salud percibida y tres Indices. La variable monoitem de salud tiene la siguiente formulación: En los 
últimos 12 meses, ¿diria que su estado de salud ha sldo muy bueno, bueno, regular, malo o muy malo?. Esta cuestión ha sido utilizada en la Encuesta Nacional de Salud (Ministerio de Sanidad y Consumo, 1997, 2003). Los índices de salud empleados han sido: el índice de molestias psicológicas que resulta de sumar las respuestas a siete ítems del cuestionario: dificultades de concentración; nerviosismo e intranquilidad; trastornos del sueño; pesadillas; miedos o fobias; cambios de humor (ánimo); humor y estado de ánimo depresivo. EI índice de molestias somáticas, formado por la suma de otros 11 f́tems que valoran molestias orgánicas: dolor de cabeza; molestias de garganta; dificultades respiratorias; temblor de manos; palpitaciones/ problemas circulatorios /mareos; diarrea; estreńimiento; molestias de estómago/ acidez; dolores de espalda y/o de columna; dolores en la nuca y/o de hombros; y molestias en el bajo vientre. Además, hemos elaborado un tercer índice que es la suma de los dos índices anteriores y que hemos denominado índice global de molestias psicosomáticas. Estos tres índices han sido formados mediante la suma de las respuestas a los ítems que componen un listado de enfermedades según la siguiente regla: $0=$ nunca, $1=$ rara vez, $2=$ algunas veces, $y$ $3=$ con frecuencia. La elaboración de los referidos índices no es una forma novedosa de proceder (Blaxter, 1990; Reig-Ferrer, CabreroGarcia, Ferrer-Cascales y Richart-Martínez, 2001) y la evidencia sobre la validez de estas escalas es más que satisfactoria ya que la consistencia interna (alfa de Cronbach) en la escala de malestar psicológico es de 0,82, en la escala de malestar físico es de 0,75 , y en el índice global de molestias psicosomáticas es de 0,85 . La correlación entre las dos escalas parciales es de $r=0,59$.

C) La calidad de vida se ha examinado a través de tres medidas. Una medida monoitem de calidad de vida general percibida: Por lo general, ¿diría que su calidad de vida es? Con cinco altemativas de respuesta que van de Muy buena a Muy mala. Es una variable que ha sido utilizada en el estudio de la calidad de vida y salud de los estudiantes universitarios (Relg-Ferrer et al., 2001). Una segunda variable que mide la calidad de vida general reciente con uno los ítems del cuestionario de COOP-Wonca, en versión adaptada por LizanTudela y Reig-Ferrer (1999). Presenta el siguiente texto: ¿Qué tal le han ido las cosas durante las últimas dos semanas?. Estupendamente, 
no podían ir mejor; Bastante bien; $A$ veces bien, a veces mal. Bien y mal a partes iguales; Bastante mal; y Muy mal, no podian haber ido peor. Finalmente, hemos utilizado la escala de Diener (1985) para medir la satisfacción vital.

D) El estrés laboral asistencial (burnout) ha sido evaluado con el Maslach Burnout Inventory -MB|- (Maslach y Jackson, 1997). Para nuestro trabajo hemos seleccionado la versión del MBI realizada por Reig-Ferrer, Cabrero-García y Ferrer-Cascales, (2003). Como ya es de sobra conocido este instrumento permite obtener tres dimensiones que definen el burnout cansancio emocional (CE), despersonalización (DP) y baja realización personal en el trabajo (RP).

E) Variables sociodemográficas: Se han utilizado como variables predictoras del mobbing la edad, el género, y la categoría académica de los profesores. Esta ultima con 6 categorías: catedráticos de universidad, titulares de universidad/ catedráticos de escuela universitaria, titulares de escuela, ayudantes de escuela y de universidad, y asociados a tiempo completo.

\section{Análisis estadístico de los datos}

La variable de respuesta mobbing se dicotomizó y se analizó bivariadamente con variables sociodemográficas, de salud y calidad de vida, y de condiciones laborales. En los casos en los que las variables explicativas eran nominales se empleó el test $c^{2}$ de Pearson, y en las restantes, ordinales, el coeficiente de correlación Rho de Spearman y el test $\chi^{2}$ de asociación lineal por lineal. Para el análisis multivariado de los determinantes laborales del mobbing se empleó la regresión logística múltiple. Se analizaron tres modelos: el primero (modelo A) reunió las siete dimensiones laborales de Warr (1999) que en el análisis bivariado mostraron una asociación estadísticamente significativa con mobbing; el segundo (modelo B), reunió los sietes índices de calidad de vida laboral de la Encuesta de Calidad de.Vida Laboral (MTAS, 2002), y el tercer modelo se compuso de los dos primeros (modelo C). Las variables se introdujeron por pasos, hacia adelante. Se presentan los valores beta, las razones de ventaja y el nivel de probabilidad de los predictores, y como medida global de los modelos el estadístico $R^{2}$ de Nagelkerke. Los análisis se han hecho con el programa SPSS 12.0. 
Tabla 1. Características sociodemograficas de los participantes en el estudio

\begin{tabular}{|c|c|}
\hline & $N^{\circ}$ de participantes (\%) \\
\hline \multicolumn{2}{|l|}{ Categoría académica } \\
\hline Catedráticos de universidad & $43(17,1 \%)$ \\
\hline Titulares de Universidad / Catedráticos de Escuela Universitaria & $68(27,9 \%)$ \\
\hline Timlares de Escuela Universitaria & $53(21 \%)$ \\
\hline Ayudantes de Universidad y Ayudantes de Escuela Universitaria & $59(23.4 \%)$ \\
\hline Asociados a Tíempo completo & $29(11,5 \%)$ \\
\hline \multicolumn{2}{|l|}{ Sexo } \\
\hline Varones & $153(60.7 \%)$ \\
\hline Mujeres & $99(39,3 \%)$ \\
\hline \multicolumn{2}{|l|}{ Edad (Media: 40,69 ; DT: 9,82 ) } \\
\hline De 22 a 35 aflos & $90(35,7 \%)$ \\
\hline De 36 a 45 afios & $84(33,3 \%)$ \\
\hline De 46 a 55 afios & $59(23,4 \%)$ \\
\hline$>$ de 55 años & $19(7,5 \%)$ \\
\hline Total de participantes en el estudio & 252 \\
\hline
\end{tabular}

\section{RESULTADOS}

Han contestado el cuestionario 252 profesores de los 409 a los que se envió la encuesta, con lo que se ha obtenido una tasa de respuesta del $61,6 \%$.

En la tabla 1 se muestran algunas características del perfil sociolaboral de los profesores. La muestra que hemos utilizado es representativa de la población de la que procede teniendo en cuenta las variables categoría académica y género.

Los datos muestran que el $22,6 \%$ de los profesores afirma haberse sentido acosado laboralmente durante el último año. Cuando se analiza la relación entre mobbing y género se advierte que ser varón o mujer no influye significativamente en referir mobbing $\left(\chi^{2}=0,246\right.$; 
$p=0,620$ ). No se ha encontrado, tampoco, que la categoría académica esté relacionada significativamente con el mobbing, aunque los datos están próximos a la significación estadística $\left(\chi^{2}=11,762 ; p=0,067\right)$. Un análisis por categorias refleja que los profesores ayudantes, tanto de escuela como de universidad $(11,4 \%)$, y los catedráticos de universidad (14\%), son los profesores que informan de una menor frecuencia de mobbing mientras que los asociados a tiempo completo $(27,6 \%)$ son los que se sienten más expuestos. Tampoco aparece una relación estadísticamente significativa entre el mobbing y la edad del profesorado $\left(\chi^{2}=36,657 ; p=0,664\right)$.

Tabla 2. Asociaciones entre la variable mobbing y las variables predictoras

\begin{tabular}{|c|c|c|c|c|c|}
\hline & $\begin{array}{c}\text { Rho de } \\
\text { Spearman }\end{array}$ & $P$ & $\begin{array}{c}x^{2} \\
\text { Jineal }\end{array}$ & g. J. & $P$ \\
\hline \multicolumn{6}{|l|}{ Variables laborides: } \\
\hline 1. Satisfucción laboral general & $-0,361$ & 0,000 & 40.648 & 1 & 0,000 \\
\hline 2. Satisfacción con la docencia & $-0,065$ & 0,304 & 3,611 & 1 & 0,304 \\
\hline 3. Satisfacción con la investigación & $-0,136$ & 0,031 & 5,980 & I & 0,014 \\
\hline 4. Sarisfaceión con los estudiantes & 0,030 & 0,631 & 0,324 & 1 & 0,569 \\
\hline 5. Satisfacción con los compafteros & $-0,483$ & 0,000 & 69,392 & 1 & 0,000 \\
\hline 6. Satisfacción situación' promoción & $-0,285$ & 0,000 & 22,844 & 1 & 0,000 \\
\hline 7. Indice de calidad de vida en el trabajo & $-0,250$ & 0,000 & 17,600 & 1 & 0,000 \\
\hline 8. Indice de condiciones de trabajo & $-0,165$ & 0,009 & 9,508 & 1 & 0.002 \\
\hline 9. İndice de orgullo en el trabajo & $-0,128$ & 0,042 & 4,164 & 1 & 0,041 \\
\hline 10. Indice de alienación en el trabajo & 0,235 & 0,000 & 15,558 & 1 & 0,000 \\
\hline 11. Indice de participación en el trabajo & $-0,352$ & 0,000 & 37,067 & 1 & 0,000 \\
\hline 12. Índice de integración en el trabajo & $-0,228$ & 0,000 & 14,528 & 1 & 0,000 \\
\hline 13. Indice de autonomía en el trabajo & $-0,321$ & 0,000 & 24,892 & 1 & 0,000 \\
\hline 14. Oportunidades de control personal & 0,198 & 0,002 & 13,460 & 1 & 0,000 \\
\hline 15. Oportunidedes para el uso de destrenas & -0.190 & 0,003 & 12,853 & 1 & 0,000 \\
\hline 16. Metas generadas extemsmente & $-0,162$ & 0,010 & 5,914 & 1 & 0,015 \\
\hline 17. Variedad en el trabajo & -0.029 & 0,643 & 0,738 & 1 & 0,390 \\
\hline 18. Claridad ambiental & $-0,119$ & 0.060 & 3,459 & 1 & 0,063 \\
\hline 19. Disponibilidad de dinero & $-0,102$ & 0,114 & 3,500 & 1 & 0,114 \\
\hline 20. Segurided fisica & $-0,189$. & 0,003 & 13,286 & 1 & 0,000 \\
\hline 21. Supervisión de apoya & $-0,454$ & 0,000 & 63,045 & 1 & 0,000 \\
\hline 22. Oportanidades para contacto interpersonal & $-0,352$ & 0,000 & 39,117 & $\mathbf{l}$ & 0,000 \\
\hline 23. Posición social valorada & $-0,144$ & 0,022 & 7,259 & 1 & 0,007 \\
\hline \multicolumn{6}{|l|}{ Variables do calidad de vida: } \\
\hline 24. Calidad de vida general & $-0,057$ & 0,371 & 1,089 & $\mathbf{l}$ & 0,207 \\
\hline 25. Calidad de vida reciente & $-0,251$ & 0,000 & 12,149 & 1 & 0,000 \\
\hline
\end{tabular}




\begin{tabular}{l|ccccc}
\hline 26. Satisfacción vital & $-0,085$ & 0,179 & 2,220 & 1 & 0,136 \\
27. Cansancio emocional (MBD) & 0,216 & 0,000 & 12,537 & 1 & 0,000 \\
28. Despersonalización (MBD) & 0,089 & 0,161 & 3,564 & 1 & 0,059 \\
29. Baja realizarión personal (MBD & $-0,002$ & 0,981 & 0,015 & 1 & 0,901 \\
Variables de salud: & & & & & \\
30. Salud percibida & $-0,011$ & 0,856 & 0,039 & 1 & 0,844 \\
31. Indice de molestias somátícas & 0,048 & 0,449 & 0,576 & 1 & 0,448 \\
32. Indice de molestias psicológices & 0,153 & 0,015 & 7,208 & 1 & 0,007 \\
33. Indice de molestlas psicosomáticas & 0,098 & 0,120 & 3,177 & 1 & 0,075 \\
Varjables sociodemogrtficas: & & & & & \\
34. Categoria académica & $-0,039$ & 0,514 & 0,428 & 1 & 0,513 \\
35. Ediad & 0,094 & 0,137 & 2,052 & 1 & 0,152 \\
36. Ginero & $0,246 *$ & 0,620 & 0,245 & 1 & 0,621 \\
\hline
\end{tabular}

${ }^{*} \chi^{2}$ de Pearson

En la tabla 2 se refleja el comportamiento relacional de la variable mobbing con otras variables relevantes de nuestro estudio sobre la calidad de vida, el trabajo y la salud de los profesores universitarios. Se observa la existencia de asociaciones significativas inversas de mayor intensidad entre el mobbing y una serie de variables laborales: satisfacción con los compañeros de trabajo (Rho $=-0,483$ ); satisfacción con el apoyo recibido por los supervisores (Rho $=-0,454$ ); satisfacción laboral en general (Rho $=-0,361)$; oportunidad para las relaciones interpersonales (Rho $=-0,352$ ) o el grado de participación en el trabajo (Rho $=-0,352$ ). De menor intensidad son las asociaciones existentes con otras variables que hemos utilizado en nuestro estudio para medir el cansancio emocional o la calidad de vida reciente. No existe asociación estadísticamente significativa entre mobbing y el estado de salud percibido por los profesores.

Los análisis de regresión logística de los determinantes laborales del mobbing han examinado tres modelos; los dos primeros son complementarios ( $A$ y $B$ ) y el tercero (modelo $C$ ) es la suma de ambos. En el modelo A (ver tabla 3) las variables explicativas introducidas en la ecuación de regresión logística han sido siete de las dimensiones que valoran satisfacción laboral: oportunidades para el control personal, 
oportunidades para el uso de destrezas, metas generadas externamente, seguridad física en el trabajo, supervisión de apoyo, oportunidad para las relaciones interpersonales, y posición social valorada (Warr, 1999). Según este modelo de regresión, los predictores del mobbing son la seguridad física y la supervisión de apoyo, con un porcentaje de explicación de la varianza de casi el $36 \%\left(R^{2}\right.$ de Nalgekerke $\left.=0,356\right)$.

El modelo $B$ incorpora los siete índices de calidad de vida laboral procedentes de las Encuestas sobre Calidad de Vida en el Trabajo (MTAS 1999, 2000, 2001, 2002): el Indice de calidad de vida en el trabajo, el índice de condiciones de trabajo, el índice de orgullo en el trabajo, el índice de alienación en el trabajo, el indice de participación en el trabajo, el índice de integración en el trabajo y el índice de autonomía en el trabajo (ver tabla 3). El índice de participación en el trabajo es el único predictor estadísticamente significativo, siendo la varianza explicada ligeramente superior al $20 \%\left(R^{2}\right.$ de Nagelkerke $\left.=0,21\right)$.

$\mathrm{El}$ modelo $\mathrm{C}$ incluye como variables explicativas las catorce variables de los modelos A y B. Sólo dos predictores explican estadísticamente la varianza del mobbing en el modelo, uno, el índice de autonomía en el trabajo, procedente del modelo $B$, y el otro, el apoyo prestado por los superiores, del modelo A. En conjunto explican el 37 de la varianza ( $R^{2}$ de Nagelkerke $=0,37$ ).

\section{DISCUSIÓN}

El primer objetivo de esta investigación ha sido determinar la presencia de mobbing en el profesorado universitario de la Universidad de Alicante. En esta línea se puede decir que prácticamente 1 de cada $4(22,6 \%)$ de estos profesores universitarios se siente objeto de mobbing. Se trata de un porcentaje algo superior al que aparece en otros estudios realizados en el medio universitario como el de Björqvist et al., (1994), quienes encuentran un $11 \%$ de profesores que refieren ser acosados entre los que sólo se dedican a la enseñanza y un $16 \%$ entre los profesores que combinan tareas docentes e investigadoras. También supera el porcentaje obtenido por Lewis (1999), quien informa que un $18 \%$ de profesores (lecturers) reconoce ser acosado psicológicamente en su trabajo. Es asimismo superior al porcentaje que aparece en el estudio de Einarsen y Skogstad 
Tabla 3. Análisis de regresión logistica entre mobbing y determinantes laborales

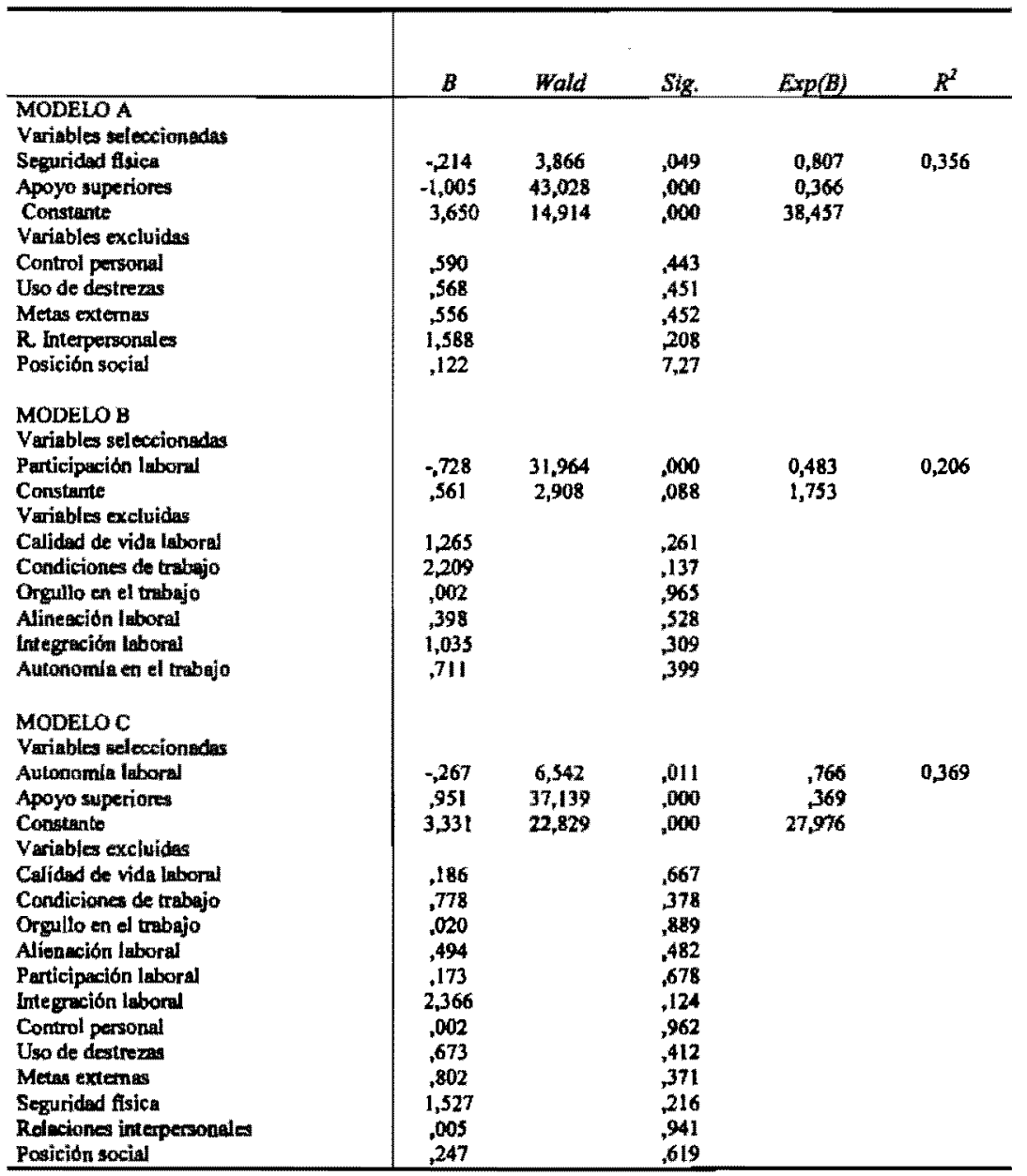


(1996), quienes con una muestra multiocupacional en la que figuran, entre otros, trabajadores universitarios, obtienen una media de $8,6 \%$ de víctimas de mobbing en los seis meses anteriores a la encuesta. Nuestros resultados se aproximan a los obtenidos por Spratlan (1995) en una universidad americana, en la que al menos un $23 \%$ de los profesores encuestados reconoce haberse sentido maltratado en su trabajo durante los 10 meses anteriores al estudio, o a los de Jennifer, Cowie y Ananiadou (2003) que utilizando una muestra multi-ocupacional en la que figuran profesores españoles (no universitarios) encuentran un $21,1 \%$ de victimas de mobbing. También se asemejan a los que aparecen en el informe CISNEROS $V$ (Piñuel y Oñate, 2004), llevado a cabo entre funcionarios de cuerpos técnicos de la administración publica española, en el que el $21 \%$ se siente objeto de acoso psicológico en el trabajo. En otro sector ocupacional, Quine (1999) halla un $38 \%$ de trabajadores sanitarios que afirman haber sufrido uno o más tipos de acoso laboral psicológico durante el año anterior al estudio. Estos resultados están próximos a los del informe CISNEROS III (Piñuel, 2002b) que arroja una incidencia de mobbing del $33 \%$ en personal de enfermería. En estos dos últimos casos la ocurrencia del mobbing es superior a la que hemos obtenido en nuestro estudio. Finalmente hay que reseñar que los informes CISNEROS I y II (Piñuel, 2001; 2002a) muestran que un $17 \%$ de trabajadores españoles de la población general refiere ser victima del mobbing, dato que resulta inferior al obtenido en nuestra investigación.

Otro de los objetivos de nuestro estudio consistía en examinar la asociación existente entre el mobbing y algunas variables sociodemográficas de interés (el género, la edad y la categoría académica del profesorado universitario). Según nuestros datos, el género del profesorado no se relaciona con el mobbing. Este resultado es coincidente con los obtenidos tanto por Einarsen y Skogstad (1996), y Fidalgo y Piñuel (2004), como por Quine (1999), y es contrario al de Björqvist et al., (1994), quienes hallan que un $24 \%$ de profesoras universitarias frente a un $17 \%$ de profesores son víctimas de acoso laboral psicológico en la universidad. También Paoli y Mellié (2001) encuentran que entre los trabajadores europeos las mujeres están algo más expuestas (un $2 \%$ más) que los varones a todas las formas de violencia laboral. 
Por otra parte, no ha aparecido relación estadísticamente significativa entre sentirse víctima del mobbing y factores como la edad y la categoría académica, en línea de resultados como el de Kivimacki et al., (2000). En relación con la edad, estudios como los de Quine (1999) y Piñuel (2001) señalan que los trabajadores más jóvenes y menos cualificados son los más expuestos al mobbing. Mientras que por el contranio, Einarsen y Skogstad (1996) afirman que son los empleados de mayor edad los que están más expuestos a este tipo de victimización laboral. Respecto de la categoría académica, Paoli y Merllíe (2001), al igual que ocurre en nuestra investigación, no hallan diferencias estadísticamente significativas en el mobbing percibido que se encuentren asociadas al estatus ocupacional del trabajador. En resumen, en nuestro trabajo la edad, el género y la categoría académica no determinan la presencia o ausencia de mobbing, si bien las diferencias en mobbing debidas a la categoría académica están próximas a la significación estadística, con una tendencia que muestra una mayor vulnerablidad ante el mobbing cuanto menor es la categoría académica del profesorado.

Finalmente, un tercer objetivo consistía en conocer los mejores predictores del mobbing. No hemos hallado estudios en los que se investigue las variables predictoras del mobbing en el medio universitario; sin embargo, entre trabajadores de la población general, Einarsen et al., (1994), Einarsen y Skoggtad (1996), Kaukanien et al., (2001), y Jennifer et al., (2003) encuentran que las condiciones de trabajo, en su conjunto, explican aproximadamente un $10 \%$ de la varianza del mobbing, mientras que la baja sattsfacción con los supervisores, el control sobre la tareas a realizar, el clima social, el conflicto de roles y especialmente los conflictos interpersonales serían los mejores predictores del mobbing. Nuestros resultados van en la línea de los previamente reseñados; los mejores predictores del mobbing que refieren los profesores universitarios son: el grado de autonomía laboral y la satisfacción con el apoyo recibido por parte de los supervisores o superiores. Estas variables explican casi un $37 \%$ de la varianza del mobbing. Hemos advertido, por el contrario, que el mobbing no parece, según nuestros resultados, tener repercusiones significativas sobre el estado de salud percibido por los profesores, sobre su valoración de la calidad de vida general, o sobre su satisfacción vital. 
Una limitación de nuestro estudio se deriva de la naturaleza transversal del diseño de investigación utilizado. Los resultados obtenidos en estudios descriptivos como el nuestro tienen una finalidad principalmente exploratoria, orientativa e incluso analítica, pero no posibilitan el establecimiento de una estricta predecibilidad, ni tampoco permiten considerar la existencia de relaciones de causalidad entre las variables que han sido examinadas en nuestra investigación. Una segunda limitación la constituye la definición operativa de mobbing que hemos empleado, que si bien conceptualmente se aproxima a la consensuada por Leyman (1990), no obstante, adolece de no ser una medida estandarizada y multiítem .

Hay que destacar, en último lugar, que nuestra evaluación del mobbing ha tenido en cuenta los criterios de frecuencia y duración que se consideran adecuados para que la medida sea válida y que se trata de uno de los primeros trabajos realizados en nuestro país en los que se estudia la presencia de mobbing y sus determinantes laborales en una muestra representativa de profesores universitarios.

\section{REFERENCIAS BIBLIOGRÁFICAS}

Björkqvist, K., Osteman, K., Hjelt-Back, M. (1994). Aggression among university employees. Aggressive Behavior № 20: 173-184.

Blaxter, M. (1990). Health and Lifestyles. Londres: Routledge.

DiMartino, V., Hoel, H., y Cooper C.L. (2003). Preventing violence and harassment in the workplace. Luxemburg: Office for Official Publications of the European Communities.

Einarsen, S, (1999). The nature and causes of bullying at work. Intemational Joumal of Manpower, Vol. 20, Ne 1/2: 16-27.

Einarsen, S. y Skogstad, A. (1996). Bullying at work: epidemiological findings in public and private organizations. European Joumal of Work and Organizational Psychology, 5:185-201.

Fidalgo, A. M. y Piñuel, I. (2004). La escala Cisneros como herramienta de valoración del mobbing. Psicothema, vol. 16: 615-624.

Jennifer, D., Cowie, H., y Ananiadou, K. (2003). Perceptions and experience of workplace bullying in five different working populations. Aggressive Behaviourvol. 29: 489-496. 
Kaukanien, A, Salmivalli, C., Björkqvist, K., Ósterman, K., Lathtinen, A., Kostamo, A., y Lagerspetz. K. (2001). Overt and covert agresion in work settings in relation to the subjetive well-being of employees. Aggressive Behaviour, vol. 27. 360-371.

Kivimäki, M., Elovainio, M, y Vathera, J. (2000). Workplace bullying and sickness absence in hospital staff. Occupational Envirommental Medecine 57: 656-660.

Kivimäki, M., Virtanen, M. Vartia, M., Elovainio, M., Vathera, J., Keltikangas-Järvinen, L. (2003). Workplace bullying and the risk of cardiovascular disease and depression. Occupational Envirommental Medecine 60:779-783.

Leymann, H. (1990). Mobbing and psychological terror at workplaces. Violence and Victims, 5: 119-126.

Lewis, D. (1999). Workplace bullying-interim findins of a study in futher and higher education in Wales. International Joumal of Manpower, Vol. 20, № 1/2: 106-118.

Lizán-Tudela, L., y Reig-Ferrer, A. (1999). Adaptación transcultural de una medida de la calidad de vida relacionada con la salud: La versión española de las viñetas COOP-Wonca. Atención Primaria, 24:7582.

Maslach, C. y Jackson, S. (1997). MBI. Inventario <<Bumout > de Maslach. Síndrome del <<quemado>> por estrés laboral asistencial. Madrid: TEA Ediciones.

Ministerio de Sanidad y Consumo (1997). Encuesta Nacional de Salud en España 1997 Madrid. Consultado el 21 de Noviembre de 2001. Accesible en: http://www.msc.es/salud/epidemiologia/ies/ encuesta.

Ministerio de Sanidad y Consumo (2003). Encuesta Nacional de Salud en España 2001 Madrid. Consultado el 5 de Diciembre de 2003. Accesible en: http://www.msc.es/salud/epidemiologia/ies/ encuesta2001/.htm

Ministerio de Trabajo y Asuntos Sociales (1999). Encuesta de Calidad de Vida en el Trabajo 1999. En: http://www.mtas.es/Estadisticas/ ECVTMelcome.htm Consultado el 21 de marzo de 2001.

Ministerio de Trabajo y Asuntos Sociales (2000). Encuesta de Calidad de Vida en el Trabajo 2000. En: http://www.mtas.es/Estadisticas/ ECVTMelcome.htm Consultado el 21 de marzo de 2001. 
Ministerio de Trabajo y Asuntos Sociales (2001). Encuesta de Calidad de Vida en el Trabajo 2001. En: http://www.mtas.es/Estadisticas/ ECVT/Welcome.htm Consultado el 25 de mayo de 2002.

Ministerio de Trabajo y Asuntos Sociales (2002). Encuesta de Calidad de Vida en el Trabajo 2002. En: http://www.mtas.es/Estadisticas/ ECVT/Welcome.htm Consultado el 3 de marzo de 2003.

Namie, G. (2000). U.S. Hostile Workplace Survey 2000. Labor Day Meeting. Benicie, September 2000. Documento accesible en la URL: http://www.bullybusters.org

Paoli, P., Merllie, D. (2001). Third European survey on working conditions 2000. Luxemburg: Office for Official Publications of the European Communities.

Pergener, T.V., Chamot, E., Etter, J.F., Richard, J.L., Gallant, S., Ricciardi, P., Iten, A., y Bumand, B. (2000). Assesment of de COOP charts with and without pictures in a Swiss population. Quality of Life Research 9: 405-414.

Piñuel, l. (2001). Mobbing. Cómo sobrevivir al acoso psicológico en el trabajo. Santander: Sal Terrae

Piñuel, I. (2002a). Informe Cisneros II. La incidencia del mobbing o acoso laboral psicológico en España. Universidad de Alcalá de Henares.

Piñuel, I. (2002b). Informe Cisneros III sobre la violencia en el entorno laboral. Presentación de los resultados preliminares de la incidencia del mobbing en los profesionales de enfermería españoles. SATSE-Universidad de Alcalá. Accesible en : www.satse.es/PDF/ AREAS_TEMATICAS/MOBBING.PDF

Piñuel, I., y Oñate, A. (2004). Informe Cisneros V. Los riesgos psicosociales en la administración. Equipo de investigación Cisneros. Universidad de Alcalá de Henares.

Quine, L. (1999). Workplace bullying in NHS community trust: staff questionnaire survey. British Medical Joumal, Vol. 18: 229-232.

Reig-Ferrer, A., Cabrero-García, J., y Ferrer-Cascales, R. I. (2003). Estrés laboral asistencial (bumout) en una muestra representativa de profesionales sanitarios de la provincia de Alicante. Alicante: Monografias del Departamento de Psicología de la Salud de la Universidad de Alicante.

Reig-Ferrer, A., Cabrero-Garcla, J., Ferrer-Cascales, R. I. y RichartMartínez, M. (2001). La calidad de vida y estado de salud de los 
estudiantes universitarios. Alicante: Publicaciones de la Universidad de Alicante.

Sáenz Barrio, O., y Lorenzo Delgado, M. (1993). La satisfacción del profesorado universitario. Granada: Monografías de Pedagogía. Universidad de Granada.

Spratlan, L. P. (1995). Interpersonal conflict wich includes mistreatment in a University workplace. Violence and Victims, 10. 285-297.

Vandekerckhove, W. y Ronald-Commers, M.S. (2003). Downward workplace mobbing: a sign of the times? Journal of Business, 45: 41-50.

Warr, P.B. (1999). Well-being and the workplace. In D. Kahneman, E. Diener y N. Swchwarz (Eds.) Wellbeing: The Foundations of Hedonic Psychology. New York: Russell Sage Foundation. 
Análisis y Modificación de Conducta, Vol. 32, № 142

Los autores del artículo "La presencia de mobbing y sus

determinantes laborales en profesores universitarios" (pp. 145-163) son J. Herranz-Bellido, A. Reig-Ferrer y J. Cabrero-García. 